\title{
Lymph Node Ratio as a Prognostic Marker in Rectal Cancer Survival: A Systematic Review and Meta-Analysis
}

\author{
Uday Karjol $^{1}$, Pavan Jonnada ${ }^{1}$, Ajay Chandranath ${ }^{1}$, Sushma Cherukuru ${ }^{2}$ \\ 1. Surgical Oncology, Kidwai Memorial Institute of Oncology, Bangalore, IND 2. Pathology, AmPath Laboratories, \\ Hyderabad, IND
}

Corresponding author: Pavan Jonnada, pavan.j.1903@gmail.com

\begin{abstract}
Introduction

The lymph node ratio (LNR) is defined as the ratio of the number of positive lymph nodes to the total number of nodes retrieved. LNR has recently emerged as a prognostic factor in rectal cancer. The objective of our study was to pool eligible studies to elucidate the prognostic role of LNR on overall survival (OS) and disease-free survival (DFS) in rectal cancer patients using a meta-analysis.
\end{abstract}

\section{Methods}

A systematic database search was performed in MEDLINE and Embase for relevant studies that reported LNR in rectal cancer. Two authors independently screened the relevant articles for selection and data extraction. As a result, a list of such studies and references, published in English up to December 2019, was obtained, and a total of 4,486 node-positive patients in 18 studies were included in this meta-analysis. RevMan software 5.3 (Cochrane Collaboration, the Nordic Cochrane Centre, Copenhagen) was used for conducting all statistical analyses.

\section{Results}

A higher LNR was significantly correlated with worse OS [hazard ratio (HR): 2.60; 95\% confidence interval (CI): $2.21-3.06$; $p \leqslant .00001]$ and DFS (HR: 2.43 ; 95\% CI: 2.11-2.80; $p \leqslant .00001$ ) in node-positive rectal cancer patients. Besides, LNR is an independent predictive and prognostic marker of OS and DFS (HR: 2.52; 95\% CI: 2.17-2.94; $p \leqslant .00001$ with $\mathrm{I}^{2}=0 \%$; $\mathrm{p}=.32$ and HR: $2.63 ; 95 \%$ CI: $2.17-3.18 ; \mathrm{p} \leqslant .00001$ with $\mathrm{I}^{2}=0 \%$; $\mathrm{p}=.63$ respectively, irrespective of lymph nodal harvest).

\section{Conclusions}

Received 04/24/2020 Review began 04/30/2020 Review ended 04/30/2020 Published 05/10/2020

\section{(c) Copyright 2020} Karjol et al. This is an open access article distributed under the terms of the Creative Commons Attribution License CC-BY 4.0., which permits unrestricted use, distribution, and reproduction in any medium, provided the original author and source are credited.
Our present study demonstrates that LNR is an independent predictor of survival in rectal cancer. LNR should be considered as a parameter in future oncological staging systems. Further well-designed randomized control trials to prospectively assess LNR as an independent predictor of rectal cancer survival are necessary before its application in daily practice.

Categories: Gastroenterology, General Surgery, Oncology

Keywords: lymph node, ratio, rectal cancer, disease free survival, overall survival, systematic review and metaanalysis

\section{Introduction}

Colorectal cancer is the third most common cancer and the second leading cause of cancer deaths globally [1]. Lymph node metastasis is considered as an important factor for predicting overall survival (OS) and disease-free survival (DFS) in non-metastatic rectal cancer [2]. Lymph node status is an essential factor in determining the need for adjuvant chemotherapy after surgical resection. The assessment of lymph node metastasis in colorectal cancer is accomplished by the tumor node metastasis (TNM) staging system. This system stages lymph node involvement according to the absolute number of the positive regional lymph nodes and recommends harvesting of at least 12 nodes. In the current staging system, rectal cancer with regional lymph node metastasis is classified as stage three, which has additional treatment adjuncts [3]. However, many reports have demonstrated a decrease in the total number of harvested lymph nodes following neoadjuvant therapy. This can lead to an underestimation of nodal staging, which may lead to false-negative nodal disease or lower nodal stage [4].

The lymph node ratio (LNR) is defined as the ratio of metastatic to the total number of harvested lymph nodes, and it has emerged as an indicator of cancer-specific survival in recent years. Berger et al. have analyzed the prognostic significance of LNR in colon cancer. They observed the data from the intergroup 
trial-0089 of adjuvant chemotherapy for stage II and III colon cancer patients and concluded that LNR is a significant factor for DFS, OS, and cancer-specific survival in patients in whom more than 10 lymph nodes were retrieved [5]. This highlighted the importance of adequate lymph node retrieval and LNR. In the present study, using a meta-analysis, we aimed to clarify the prognostic role of LNR in patients with nodepositive rectal cancer. To that end, we examined the relationship of LNR with OS and DFS in such patients.

\section{Materials And Methods}

\section{Search strategy}

We performed a systematic literature search on MEDLINE, Embase, and Google Scholar databases for articles published before January 2020 using the following strategy: articles were searched using Medical Education Subject Headings (MeSH) keywords "lymph node" AND "ratio" AND "rectal cancer" OR "rectal carcinoma" AND "node-positive" OR "metastatic lymph node." Preferred Reporting Items for Systematic Reviews and Meta-Analyses (PRISMA) guidelines were followed for searching and reporting of articles.

\section{Study selection}

All studies that reported an association of LNR with OS and DFS for rectal cancer patients were identified by a comprehensive computer-based search. Two authors (PJ and UK) independently assessed titles and abstracts for eligibility. We scanned the reference lists of articles for similar additional articles. All the screened articles were assessed for eligibility, and any disagreement was resolved through discussion. We included studies in the meta-analysis if the following criteria were met: studies that were published in English, studies that were clinical trials, studies that compared the survival of rectal cancer based on LNR, and studies that included quantitative outcome data after multivariate analysis [hazard ratio (HR) for OS and DFS]. The exclusion criteria were as follows: inability to extract data from the published results; studies containing republished data; publications in the form of editorials, comments, review articles, meeting abstracts, or those which excluded reported outcomes.

\section{Data extraction}

Two authors (PJ and UK) independently extracted relevant data from the screened full-text articles. For each study that fulfilled the criteria for inclusion, the data extracted include the following: the basic characteristics of the study including the name of the first author, year of publication, study setting, design of the study, duration of the study, data sources, and multivariate adjustments; the basic patient characteristics including age, gender, stage, treatment, and survival periods; comparative outcomes, including HR for OS, DFS, and recurrence on different LNR subgroups.

\section{Quality assessment}

Two authors (PJ and AC) independently appraised the quality of each included study using the NewcastleOttawa scale. The details of the included studies are shown in Table 1 [6-23]. A study was considered of poor quality if it did not meet more than one criterion in the selection domain, if there was no score in the compatibility domain, and if it did not meet more than one of the criteria in the outcome domain. Any disagreements between reviewers were resolved by consensus. 


\section{Cureus}

\begin{tabular}{|c|c|c|c|c|c|c|c|c|c|c|c|c|c|}
\hline \multirow{2}{*}{$\begin{array}{l}\text { Author name } \\
\text { Peng et al. [6] }\end{array}$} & \multirow{2}{*}{$\begin{array}{l}\text { Year } \\
2008\end{array}$} & \multirow{2}{*}{$\begin{array}{l}\text { Study } \\
\text { design }\end{array}$} & \multirow{2}{*}{$\begin{array}{l}\begin{array}{l}\text { Sample } \\
\text { size }\end{array} \\
318\end{array}$} & \multirow{2}{*}{$\begin{array}{l}\text { NACRT } \\
\text { No }\end{array}$} & \multirow{2}{*}{$\begin{array}{l}\text { No. of average } \\
\text { nodes } \\
12\end{array}$} & \multicolumn{2}{|c|}{ Endpoints } & \multirow{2}{*}{$\begin{array}{l}\text { Median follow-up } \\
\text { (months) } \\
41\end{array}$} & \multirow{2}{*}{$\begin{array}{l}\text { NOS } \\
7 \\
7\end{array}$} & \multicolumn{4}{|c|}{ LNR stratification } \\
\hline & & & & & & os & DFS & & & $<0.14$ & $\begin{array}{l}0.14- \\
0.49\end{array}$ & $>0.49$ & - \\
\hline Kim et al. [7] & 2009 & RCS & 421 & No & 17 & os & - & 53 & 7 & $<0.1$ & $<0.2$ & $<0.4$ & $>0.4$ \\
\hline Dekker et al. [8] & 2010 & RCS & 605 & Yes & 9 & OS & DFS & 120 & 7 & $<0.6$ & $>0.6$ & - & - \\
\hline Kang et al. [9] & 2011 & RCS & 75 & Yes & 18 & OS & - & 35.1 & 7 & $<0.143$ & $>0.143$ & - & - \\
\hline $\begin{array}{l}\text { Kobayashi et al. } \\
\text { [10] }\end{array}$ & 2011 & RCS & 452 & No & 17 & OS & - & NA & 8 & $<0.04$ & $\begin{array}{l}0.04- \\
0.07\end{array}$ & $\begin{array}{l}0.08- \\
0.15\end{array}$ & $\begin{array}{l}0.15- \\
1\end{array}$ \\
\hline Allaix et al. [11] & 2012 & PCS & 129 & Yes & 12 & os & DFS & 122 & 7 & $\begin{array}{l}0.01- \\
0.25\end{array}$ & $>0.25$ & - & - \\
\hline Lee et al. [12] & 2012 & PCS & 519 & Yes & 15 & OS & DFS & 52 & 7 & $<0.15$ & $\begin{array}{l}0.16- \\
0.3\end{array}$ & $>0.3$ & - \\
\hline $\begin{array}{l}\text { Madobouly et al. } \\
\text { [13] }\end{array}$ & 2013 & PCS & 115 & Yes & 12 & os & DFS & 37 & 6 & $<0.375$ & $>0.375$ & - & - \\
\hline $\begin{array}{l}\text { La lorre et al. } \\
\text { [14] }\end{array}$ & 2013 & PCS & 508 & Yes & 15 & os & DFS & 50 & 8 & $<0.2$ & $>0.2$ & - & - \\
\hline $\begin{array}{l}\text { Nadoshan et al. } \\
\text { [15] }\end{array}$ & 2013 & PCS & 128 & Yes & 10 & os & DFS & 39 & 8 & $<0.2$ & $>0.2$ & - & - \\
\hline $\begin{array}{l}\text { Junginger et al. } \\
\text { [16] }\end{array}$ & 2014 & PCS & 237 & Yes & NA & os & DFS & 55 & 8 & $<0.1$ & $<0.2$ & $<0.3$ & $>0.3$ \\
\hline Zeng et al. [17] & 2014 & PCS & 131 & Yes & 14 & os & DFS & 49 & 8 & $<0.2$ & $>0.2$ & - & - \\
\hline Koo et al. [18] & 2015 & RCS & 125 & Yes & 17 & os & DFS & 55 & 8 & $<0.15$ & $>0.15$ & - & - \\
\hline Park et al. [19] & 2015 & RCS & 967 & Yes & 16.5 & - & DFS & 40 & 8 & $<0.25$ & $>0.25$ & - & - \\
\hline $\begin{array}{l}\text { Leonard et al. } \\
\text { [20] }\end{array}$ & 2016 & RCS & 357 & Yes & 13 & os & DFS & NA & 7 & $<0.2$ & $>0.2$ & - & - \\
\hline Zuo et al. [21] & 2016 & RCS & 264 & Yes & 11 & OS & DFS & 45 & 7 & $<0.2$ & $>0.2$ & - & - \\
\hline $\begin{array}{l}\text { Fritzmann J et al. } \\
\text { [22] }\end{array}$ & 2018 & PCS & 630 & Yes & 15 & os & - & 36.1 & 7 & $\begin{array}{l}<0.01- \\
0.17\end{array}$ & $\begin{array}{l}0.18- \\
0.41\end{array}$ & $\begin{array}{l}0.42- \\
0.69\end{array}$ & $>0.69$ \\
\hline Chen et al. [23] & 2018 & RCS & 133 & Yes & 12 & os & DFS & 40 & 7 & $<0.15$ & $>0.15$ & - & - \\
\hline
\end{tabular}

\section{TABLE 1: Characteristics of the included studies}

NACRT: neoadjuvant chemoradiotherapy; NOS: Newcastle-Ottawa score; DFS: disease-free survival; NA: not available; OS: overall survival; PCS: prospective cohort study; RCS: retrospective cohort study

\section{Statistical analysis}

The statistical analysis was performed using RevMan software, version 5.3 (Cochrane Collaboration, the Nordic Cochrane Centre, Copenhagen). Continuous variables were analyzed by the HR, and $95 \% \mathrm{CI}$ was recorded. Heterogeneity was assessed using $\chi 2$ and $\mathrm{I}^{2}$ tests. $\mathrm{I}^{2}$ of $0-40,30-60,50-70$, and $>75 \%$ represent low, moderate, substantial, and considerable heterogeneity, respectively. Studies with a $\mathrm{p}$-value of $<.1$ and $\mathrm{I}^{2}$ indicated substantial heterogeneity. A random-effects model was used to estimate the pooled HR if significant heterogeneity existed in the fixed-effects model. Otherwise, the fixed-effects model was used with $\mathrm{p}>.10$ and $\mathrm{I}^{2}<25 \%$. The $\mathrm{z}$-test was used to determine the pooled HR, and the significance was set to reject the null hypothesis at $\mathrm{p}<.05$. Funnel plots were undertaken to investigate possible bias.

\section{Results}

\section{Studies included}


A total of 422 potentially relevant articles were identified with our predefined search strategy. Based on inclusion and exclusion criteria and following the screening of titles and abstracts, 367 studies were excluded. After excluding duplicates, the reviewers identified 31 studies for an extensive review. Of these, 18 studies were entered into meta-analysis after the exclusion of 24 studies (Figure 1). The quality of articles as assessed by the Newcastle-Ottawa score was by and large acceptable. The main characteristics of the included studies are provided in Table 1.

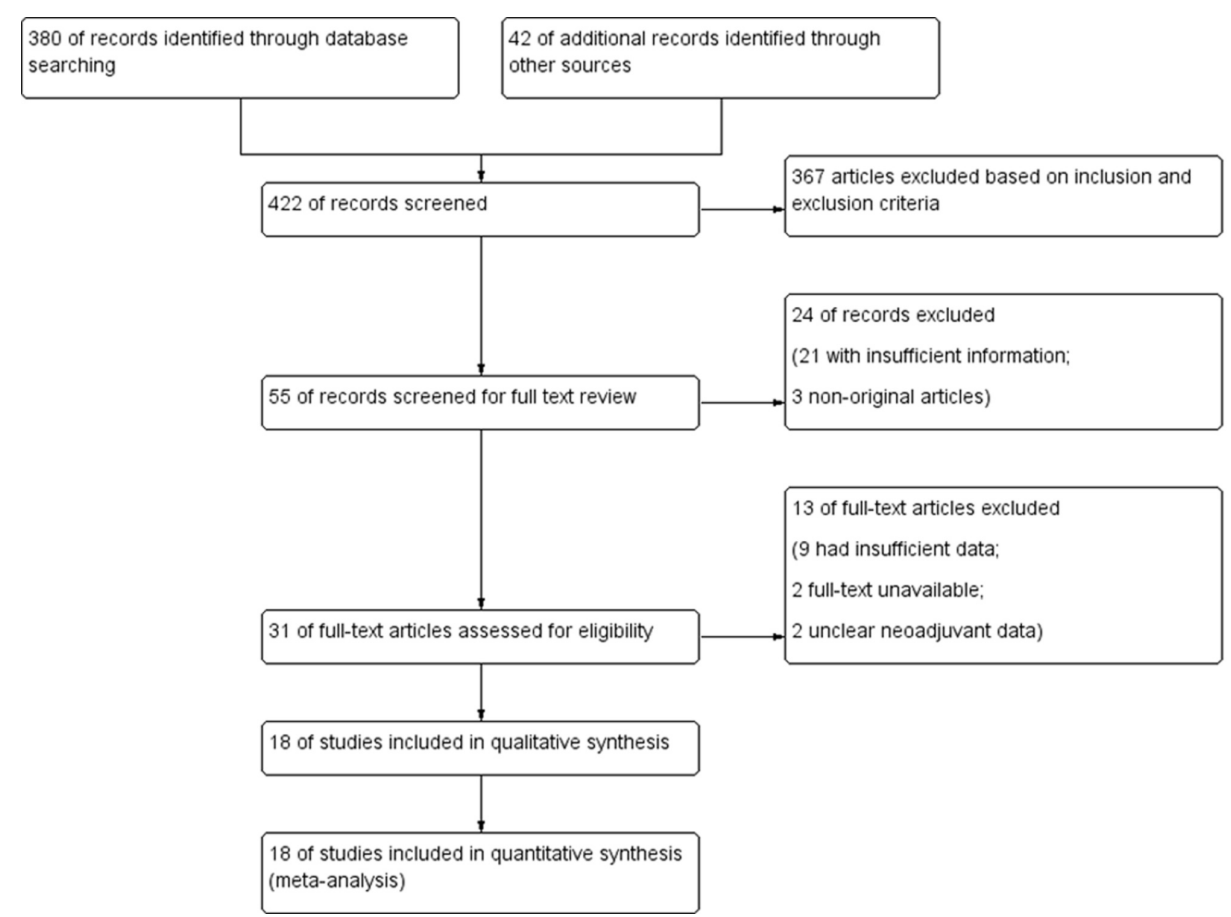

FIGURE 1: PRISMA flow chart showing study selection

PRISMA: Preferred Reporting Items for Systematic Reviews and Meta-Analyses

\section{Meta-analysis results}

The estimated mean number of harvested lymph nodes was $12.9 \pm 1.03$ in cases overall. In the present study, we performed the meta-analysis and examined the effect of LNR on OS and DFS. OS, as the primary outcome, was extracted from 17 studies with available data. A pooled HR and its $95 \%$ confidence interval (CI) were calculated with a fixed model for OS (Figure 2) [6,8,11-21,23]. The result showed that high LNR predicts poor OS. The pooled HR was 2.52 (95\% CI: $2.20-2.88$ ) for OS with a statistically significant p-value of $<.00001$. Insignificant heterogeneity was found $\left(\mathrm{I}^{2}=18 \%\right.$ and $\left.\mathrm{p}=.24\right)$ on the fixed-effects model. Among these studies, 11 reported LNR with a single cut-off value and pooled HR of 2.64 (95\% CI: 2.13-3.27; p<.00001).

Insignificant heterogeneity was found $\left(\mathrm{I}^{2}=11 \%\right.$ and $\left.\mathrm{p}=.34\right)$ on the fixed-effects model. Among these studies, five used 0.2 , two used 0.15 , one used 0.14 , one used 0.375 , and one study used 0.6 and reported a pooled HR of 2.50 (95\% CI: 2.13-2.94; $\mathrm{p}<.00001$ ) with little heterogeneity $\left(\mathrm{I}^{2}=16 \%, \mathrm{p}=.30\right)$. Two studies reported LNR with two cut-off values subdividing the patients into low risk, medium risk, and high-risk LNR groups, and they reported pooled HR of 4.32 ( $95 \%$ CI: $2.52-7.41 ; \mathrm{p}<.00001$ ). Minor heterogeneity was found $\left(\mathrm{I}^{2}=0 \%\right.$ and $\mathrm{p}=.35$ ). Four studies reported three cut-off values, and they reported polled HR of 2.25 (95\% CI: 1.72-2.93; $\mathrm{p}<.00001)$, with minimal heterogeneity $\left(\mathrm{I}^{2}=0 \%, \mathrm{p}=.51\right)$. Neoadjuvant therapy was given before surgery in 14 studies, and they reported pooled HR of 2.70 ( $95 \%$ CI: $2.18-3.34$ ) with $\mathrm{I}^{2}=30 \%$ and $\mathrm{p}=.14$. Thirteen studies reported retrieval of more than 12 nodes with pooled HR of 2.78 ( $95 \%$ CI: $2.18-3.55$ ) with $\mathrm{I}^{2}=24 \%$ and $\mathrm{p}=.21$, and three studies reported retrieval of 12 or more nodes with pooled HR of 2.37 (95\% CI: 1.95-2.89), as shown in Table 2. It was demonstrated that reported retrieval of more than 12 nodes and less than 12 nodes showed an overall pooled HR of 2.52 (95\% CI: $2.17-2.94 ; \mathrm{p} \leqslant .00001)$ with $\mathrm{I}^{2}=0 \%$ and $\mathrm{p}=.32$. 


\section{Cureus}

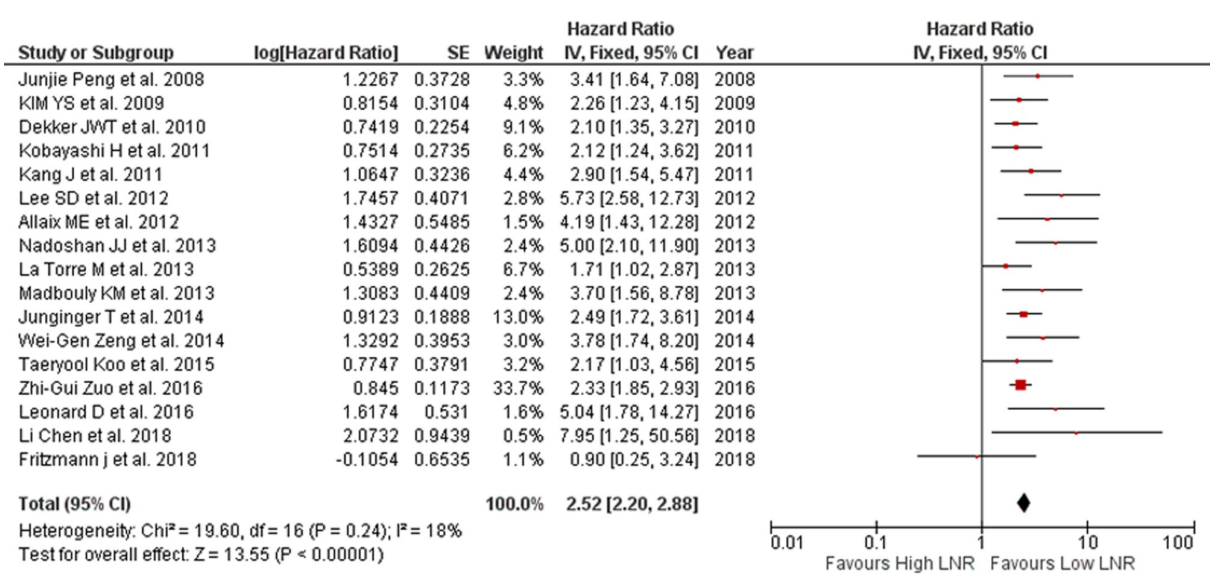

\section{FIGURE 2: Forest plot showing LNR and OS}

OS: overall survival; LNR: lymph node ratio; SE: standard error; $\mathrm{CI}$ : confidence interval

\begin{tabular}{|c|c|c|c|c|c|c|c|c|}
\hline \multirow{2}{*}{\multicolumn{2}{|c|}{ Subgroups }} & \multicolumn{4}{|c|}{ Pooled estimates } & \multicolumn{3}{|c|}{ Heterogeneity } \\
\hline & & No.of studies & HR & $95 \% \mathrm{Cl}$ & P-value & Model & $\mathrm{I}^{2} \%$ & P-value \\
\hline Overall & & 17 & 2.52 & $2.20-2.88$ & & Fixed & $18 \%$ & 0.24 \\
\hline \multirow[t]{2}{*}{ No.of nodes } & $<12$ & 3 & 2.37 & $1.95-2.89$ & & Fixed & $37 \%$ & 0.21 \\
\hline & $\geq 12$ & 13 & 2.78 & 2.18-3.55 & & Fixed & $24 \%$ & 0.21 \\
\hline \multirow[t]{2}{*}{ CTRT } & Yes & 14 & 2.70 & 2.18-3.34 & & Fixed & $30 \%$ & 0.14 \\
\hline & No & 3 & 2.50 & $1.88-3.31$ & & Fixed & $0 \%$ & 0.59 \\
\hline \multirow[t]{4}{*}{ LNR cut-off } & 0.1 & 2 & 3.25 & $1.74-6.09$ & .00001 & Fixed & $2 \%$ & 0.31 \\
\hline & 0.2 & 6 & 2.61 & $1.93-3.54$ & & Random & $36 \%$ & 0.17 \\
\hline & 0.3 & 2 & 4.69 & 2.61-8.42 & & Fixed & $0 \%$ & 0.47 \\
\hline & 0.6 & 2 & 1.92 & $1.26-2.91$ & .002 & Fixed & $33 \%$ & 0.22 \\
\hline
\end{tabular}

\section{TABLE 2: Data for LNR and OS}

Cl: confidence interval; CTRT: chemoradiation; HR: hazard ratio; LNR: lymph node ratio; OS: overall survival

DFS, as the primary outcome, was extracted from 14 studies with available data. A pooled HR and its $95 \% \mathrm{CI}$ were calculated with a fixed model for OS (Figure 3). The result showed that low LNR is associated with improved DFS, and high LNR predicts poor DFS. The pooled HR was 2.43 (95\% CI: 2.11-2.80) for DFS with a statistically significant $\mathrm{p}$-value of $<.00001$. Insignificant heterogeneity was found $\left(\mathrm{I}^{2}=0 \%\right.$ and $\left.\mathrm{p}=.46\right)$ on the fixed-effects model. Among these studies, nine reported LNR with a single cut-off value and pooled HR of 2.86 (95\% CI: 2.26-3.62; $\mathrm{p}<.00001)$. Insignificant heterogeneity was found $\left(\mathrm{I}^{2}=0 \%\right.$ and $\mathrm{p}=.64$ ) on the fixedeffects model. Two studies reported LNR with two cut-off values subdividing the patients into low risk, moderate risk, and high-risk LNR groups, and they reported pooled HR of 3.27 (95\% CI: 1.94-5.52; $\mathrm{p}<.00001)$. Minor heterogeneity was found $\left(\mathrm{I}^{2}=0 \%\right.$ and $\left.\mathrm{p}=.49\right)$. Neoadjuvant therapy was given before surgery in 14 studies, and they reported pooled HR of 2.79 ( $95 \%$ CI: 2.25-3.47) with $\mathrm{I}^{2}=0 \%$ and $\mathrm{p}=.49$. Thirteen studies reported retrieval of more than 12 nodes with pooled HR of 2.38 (95\% CI: $2.01-2.80$ ) with $\mathrm{I}^{2}=21 \%$ and $\mathrm{p}=.25$, and three studies reported retrieval of 12 or more nodes with pooled HR of 2.90 (95\% CI: $1.85-4.54$ ), as shown in Table 3. It was demonstrated that reported retrieval of more than 12 nodes and less than 12 nodes showed an overall pooled HR of 2.63 (95\% CI: 2.17-3.18; $p \leqslant .00001$ ) with $\mathrm{I}^{2}=0 \%$ and $\mathrm{p}=.63$. 


\section{Cureus}

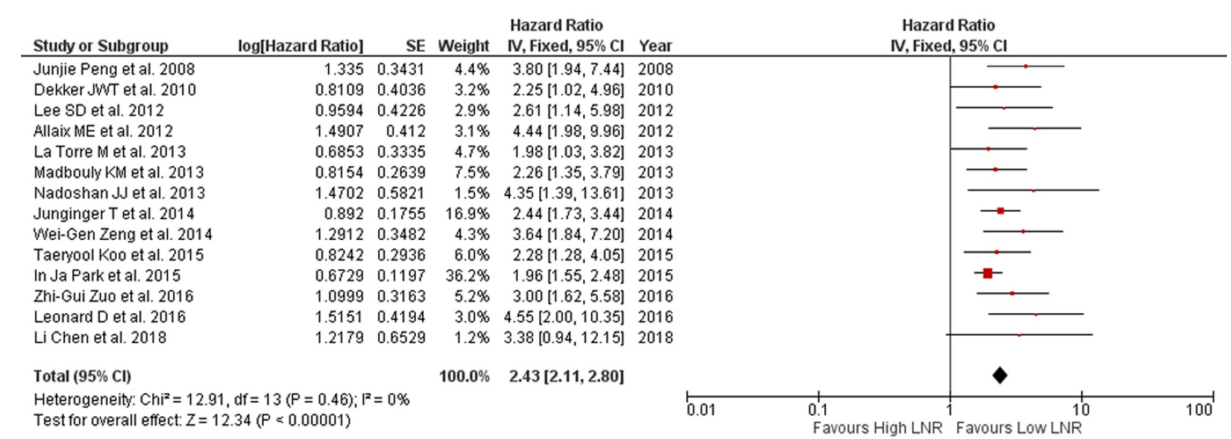

\section{FIGURE 3: Forest plot showing LNR and DFS}

DFS: disease-free survival; LNR: lymph node ratio; SE: standard error; CI: confidence interval

\begin{tabular}{|c|c|c|c|c|c|c|c|c|}
\hline \multirow{2}{*}{ Subgroups } & & \multicolumn{4}{|c|}{ Pooled estimates } & \multicolumn{3}{|c|}{ Heterogeneity } \\
\hline & & No.of studies & HR & $95 \%$ Cl & P-value & Model & $1^{2} \%$ & P-value \\
\hline Overall & & 14 & 2.43 & $2.11-2.80$ & & Fixed & $0 \%$ & 0.46 \\
\hline \multirow[t]{2}{*}{ No.ot nodes } & $<12$ & 3 & 2.90 & $1.85-4.54$ & & Fixed & $0 \%$ & 0.64 \\
\hline & $\geq 12$ & 13 & 2.38 & $2.01-2.80$ & & Fixed & $21 \%$ & 0.25 \\
\hline \multirow[t]{2}{*}{ CIRI } & Yes & 14 & 2.79 & $2.25-3.47$ & & Fixed & $0 \%$ & 0.78 \\
\hline & No & 3 & 2.34 & $1.75-3.15$ & & Random & $48 \%$ & 0.15 \\
\hline \multirow[t]{3}{*}{ LNR cut-off } & 0.1 & 2 & 1.99 & $1.58-2.51$ & .00001 & Fixed & $0 \%$ & 0.41 \\
\hline & 0.2 & 6 & 2.89 & 2.17-3.84 & & Fixed & $0 \%$ & 0.54 \\
\hline & 0.3 & 2 & 2.35 & $1.52-3.65$ & & Fixed & $0 \%$ & 0.77 \\
\hline
\end{tabular}

\section{TABLE 3: Data for LNR and DFS}

Cl: confidence interval; CTRT: chemoradiation; HR: hazard ratio; LNR: Iymph node ratio; DFS: disease-free survival

\section{Publication bias}

The publication bias of the included studies was evaluated by funnel plots. No visual publication bias was established, as shown in Figure 4 and Figure 5 [6-18,20-23]. This indicated that the publication bias was small in the current meta-analysis. 


\section{Cureus}

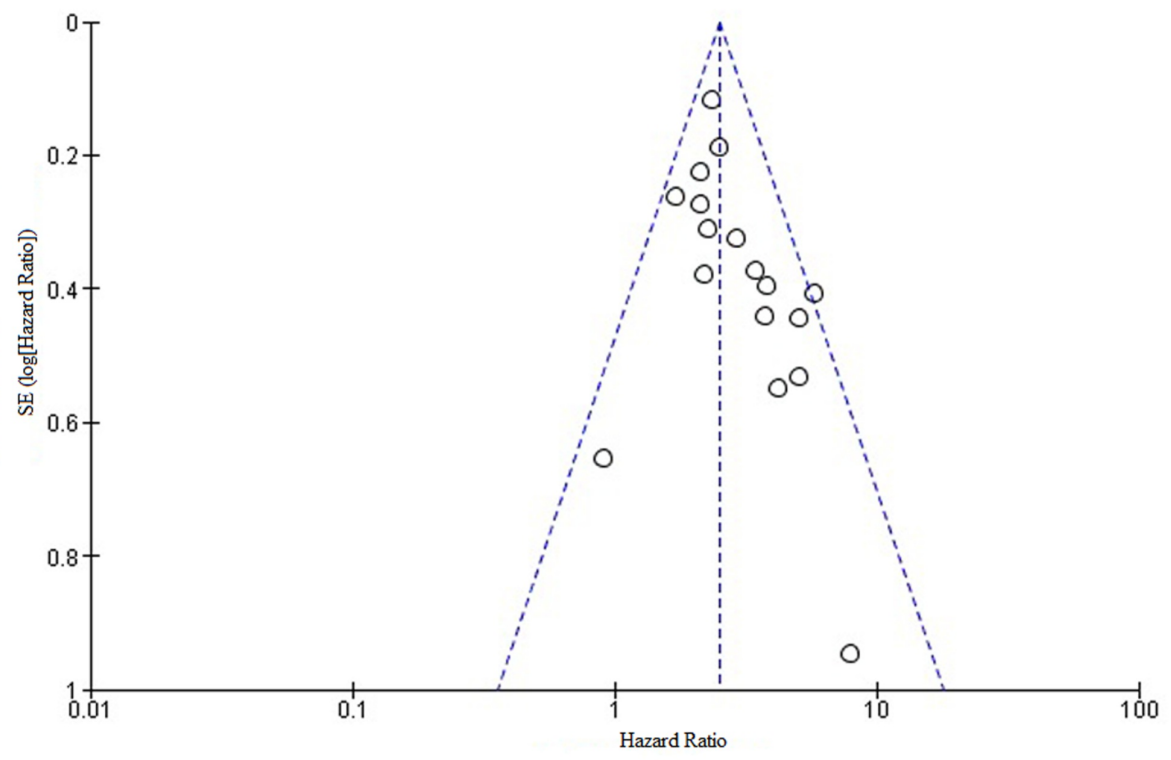

FIGURE 4: Funnel plot showing LNR and OS

OS: overall survival; LNR: lymph node ratio; SE: standard error

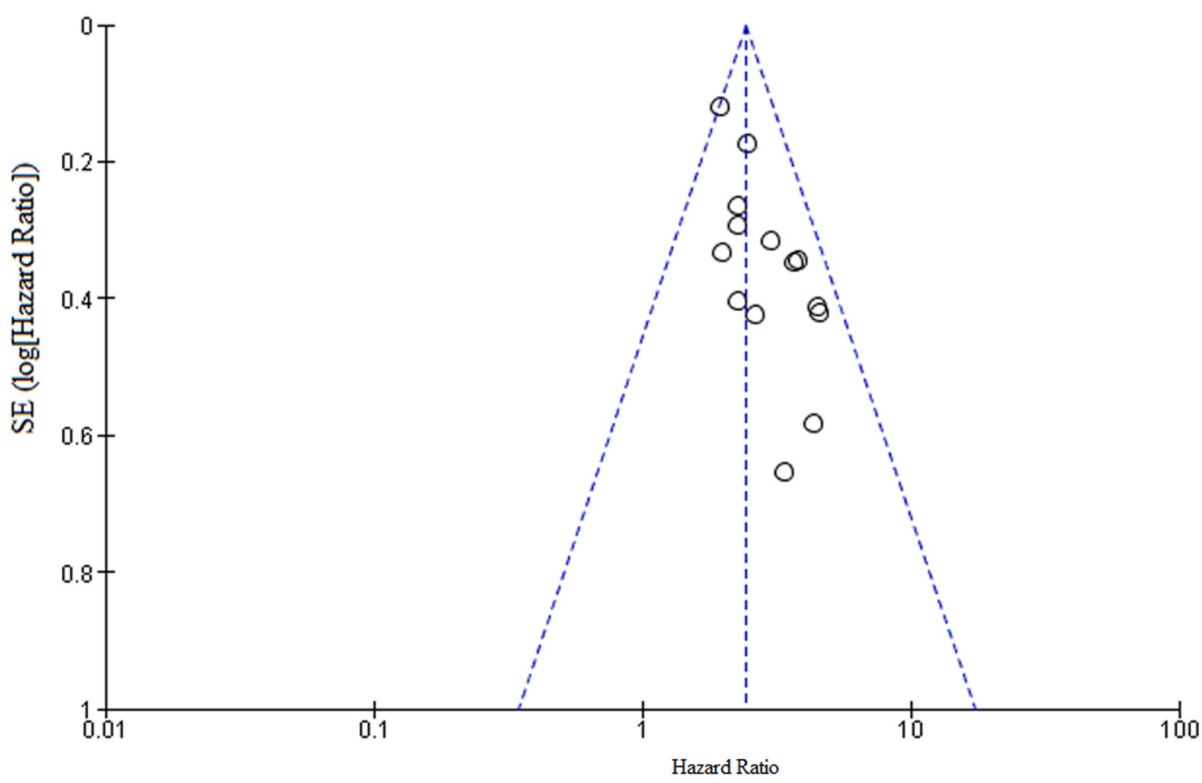

FIGURE 5: Funnel plot showing LNR and DFS

DFS: disease-free survival; LNR: lymph node ratio; SE: standard error

\section{Discussion}

Lymph nodal metastasis is an essential mechanism involved in the spread of cancers. Quantitative evaluation of the lymph nodal burden has been validated as a powerful prognostic indicator in patients with rectal cancer. Moreover, the absolute number of positive nodes has been recognized as an influential prognostic marker of adverse outcomes. It has been shown that prognosis worsens with the increasing number of metastatic lymph nodes (MLN) [24]. Hence, in the current American Joint Committee on Cancer (AJCC) staging system, the nodal disease is categorized as the $\mathrm{N}$ category and is stratified on the basis of the number of MLNs. The N category is further divided into N1 (1-3 MLN) and N2 (>4 MLN) [3]. Although this system has been shown to predict the long-term outcomes with good accuracy, it is well known that TNM 
does not consider a few other important features regarding lymph node metastasis. Nonetheless, studies have reported that a higher number of negative lymph nodes were independently associated with improved survival in patients with colorectal cancer [25]. To invalidate the limitation of the N stage, LNR has been studied. Our meta-analysis demonstrated the role of LNR in the prognostication of rectal cancer patients. Our pooled results indicate that higher LNR is associated with worse OS and DFS. The finding of low heterogeneity across studies has further added durability to the results.

Fielding et al. suggested that examination of at least 12 lymph nodes as an evaluation of less than that of suggested numbers led to a high false-negative rate of lymph node metastasis and under-staging [26]. The current TNM staging system also recommends the evaluation of 12 lymph nodes to ascertain the proper stage [3]. The number of examined lymph nodes has been reported to be influenced by patient-related factors such as location, stage, and use of neoadjuvant treatment, along with surgical and pathological factors. However, with the emerging interest in the implementation of neoadjuvant chemoradiotherapy (NACRT) and especially its consequent impact on lymph node yield retrieval, controversy still exists regarding the absolute lymph node yield. It is acknowledged that the absolute number of retrieved lymph nodes would significantly reduce with preoperative chemoradiation [27]. This has led to the implementation of LNR to solve the limitations associated with the N category of the TNM staging system.

Some studies have stated that LNR has a significant influence on survival in patients only when the examined rate of lymph nodes is greater than 10-12 [28]. This has raised concern regarding the utility of LNR over the traditional $\mathrm{N}$ category of the TNM staging system. Our study results demonstrated that higher LNR is associated with worse OS and DFS and this association remained significant, irrespective of nodal status in rectal cancer patients. These findings were further supported by a recent meta-analysis of 33 studies that included a total of 75,839 patients with node-positive colorectal cancer. In this study by Zhang et al., high LNR was significantly associated with low OS (HR: 1.91; 95\% CI: 1.71-2.14; p<.001) and DFS (HR: 2.75; 95\% CI: $2.14-3.53 ; \mathrm{p}<.001)$. They also reported that LNR remained a significant prognostic factor regardless of the number of harvested nodes and reported an HR of 1.97, 95\% CI of 1.71-2.26, and p-value of <.001 for the subgroup with more than 12 harvested lymph nodes, and an HR of 1.74, 95\% CI of 1.40-2.17, and p-value of $<.001$ for the subgroup with less than 12 harvested lymph nodes [29].

In the current meta-analysis, we looked for the studies that reported outcomes of patients who underwent NACRT because it has been reported that the total number of retrieved lymph nodes and positive lymph nodes may decrease after chemoradiation [27]. However, our study demonstrated that though neoadjuvant therapy was used in 14 studies, a lymph nodal yield of greater than 12 was observed in 13 studies. Also, our study demonstrated no difference between those with less than 12 lymph nodes and those with more than 12 lymph nodes regarding OS and DFS. These findings are supported by a recent study that demonstrated that though NACRT reduces the lymph nodal yield, it still has no significance on the survival of the patient [30].

The strengths of this meta-analysis are the precision of estimates that are based on a large dataset. This meta-analysis included 18 studies involving 4,486 node-positive rectal cancer patients. The statistical power is satisfactory enough for our results. The other strengths of this meta-analysis are the precision of LNRspecific estimates and the investigation of many covariates. The cut-off value of LNR in each included study is different altogether. The most reliable cut-off value for defining LNR, which could predict the prognosis of rectal cancer patients, is a subject of debate. However, in our study, there is significant statistical power with little heterogeneity when a cut-off of less than 0.2 is examined in a single cut-off stratifying system. However, a large cohort study or an individual patient data meta-analysis is required to justify our results and ascertain infinitesimal differences. Finally, the other strength of our meta-analysis is the minimal heterogeneity between studies and their subgroups, which enhances the robustness of the results.

Our findings should be interpreted within the structure of the effectiveness and limitations of a study-level meta-analysis of heterogeneous studies. There are certain limitations in our study that need to be spelled out. First, there was the inclusion of retrospective studies; therefore, there exists a possibility of unavoidable selection bias. Secondly, in the background of varied cut-off values of LNR generated through various methods, the heterogeneity analysis demonstrated homogeneity. This could have been responsible for likely pooling of these LNR cut-offs in the analysis that was done to predict OS and DFS. And, finally, the surgical and pathological qualities vary among different medical centers in which these studies were conducted.

\section{Conclusions}

Our meta-analysis reviewed the current research targeting the prognostic role of LNR in assessing survival in rectal cancer patients. Our findings have demonstrated that a higher LNR is a predictor of poor OS and DFS. Additionally, our study has demonstrated that LNR is an independent prognostic marker for assessing OS and DFS, irrespective of NACRT and lymph nodal harvest. We conclude that the LNR could provide answers for the lacunae in the N category of the current TNM staging system. 


\section{Additional Information \\ Disclosures}

Human subjects: All authors have confirmed that this study did not involve human participants or tissue. Animal subjects: All authors have confirmed that this study did not involve animal subjects or tissue. Conflicts of interest: In compliance with the ICMJE uniform disclosure form, all authors declare the following: Payment/services info: All authors have declared that no financial support was received from any organization for the submitted work. Financial relationships: All authors have declared that they have no financial relationships at present or within the previous three years with any organizations that might have an interest in the submitted work. Other relationships: All authors have declared that there are no other relationships or activities that could appear to have influenced the submitted work.

\section{References}

1. Bray F, Ferlay J, Soerjomataram I, Siegel RL, Torre LA, Jemal A: Global cancer statistics 2018: GLOBOCAN estimates of incidence and mortality worldwide for 36 cancers in 185 countries. CA Cancer J Clin. 2018, 68:394-424. 10.3322/caac.21492

2. Gunderson LL, Jessup JM, Sargent DJ, Greene FL, Stewart AK: Revised TN categorization for colon cancer based on national survival outcomes data. J Clin Oncol. 2010, 28:264-71. 10.1200/JCO.2009.24.0952

3. Jessup J, Goldberg R, Asare E, et al.: Colon and rectum. AJCC Cancer Staging Manual. Amin MB, Edge S, Greene F, et al. (ed): Springer, New York City, NY; 2017. 8:

4. Huang B, Feng Y, Ni M, Chen C, Cai G: Heterogeneous survival between stage IIA and stage IIIA colon cancer when different numbers of lymph nodes are harvested. ANZ J Surg. 2018, 88:316-21. 10.1111/ans.13737

5. Berger AC, Sigurdson ER, LeVoyer T, et al.: Colon cancer survival is associated with decreasing ratio of metastatic to examined lymph nodes. J Clin Oncol. 2005, 23:8706-12. 10.1200/JCO.2005.02.8852

6. Peng J, Xu Y, Guan Z, et al.: Prognostic significance of the metastatic lymph node ratio in node-positive rectal cancer. Ann Surg Oncol. 2008, 15:3118-23. 10.1245/s10434-008-0123-8

7. Kim YS, Kim JH, Yoon SM, et al.: Lymph node ratio as a prognostic factor in patients with stage III rectal cancer treated with total mesorectal excision followed by chemoradiotherapy. Int J Radiat Oncol Biol Phys. 2009, 74:796-802. 10.1016/j.ijrobp.2008.08.065

8. Dekker JW, Peeters KC, Putter H, Vahrmeijer AL, van de Velde CJ: Metastatic lymph node ratio in stage III rectal cancer; prognostic significance in addition to the 7th edition of the TNM classification. Eur J Surg Oncol. 2010, 36:1180-6. 10.1016/j.ejso.2010.09.007

9. Kang J, Hur H, Min BS, Lee KY, Kim NK: Prognostic impact of the lymph node ratio in rectal cancer patients who underwent preoperative chemoradiation. J Surg Oncol. 2011, 104:53-8. 10.1002/jso.21913

10. Kobayashi H, Mochizuki H, Kato T, et al.: Lymph node ratio is a powerful prognostic index in patients with stage III distal rectal cancer: a Japanese multicenter study. Int J Colorectal Dis. 2011, 26:891-6. 10.1007/s00384-011-1173-0

11. Allaix ME, Arezzo A, Cassoni P, Mistrangelo M, Giraudo G, Morino M: Metastatic lymph node ratio as a prognostic factor after laparoscopic total mesorectal excision for extraperitoneal rectal cancer. Surg Endosc. 2013, 27:1957-67. 10.1007/s00464-012-2694-5

12. Lee SD, Kim TH, Kim DY, et al.: Lymph node ratio is an independent prognostic factor in patients with rectal cancer treated with preoperative chemoradiotherapy and curative resection. Eur J Surg Oncol. 2012, 38:478-83. 10.1016/j.ejso.2012.03.002

13. Madbouly KM, Abbas KS, Hussein AM: Metastatic lymph node ratio in stage III rectal carcinoma is a valuable prognostic factor even with less than 12 lymph nodes retrieved: a prospective study. Am J Surg. 2014, 207:824-31. 10.1016/j.amjsurg.2013.07.022

14. La Torre M, Mazzuca F, Ferri M, et al.: The importance of lymph node retrieval and lymph node ratio following preoperative chemoradiation of rectal cancer. Colorectal Dis. 2013, 15:e382-8. 10.1111/codi.12242

15. Nadoshan JJ, Omranipour R, Beiki O, Zendedel K, Alibakhshi A, Mahmoodzadeh H: Prognostic value of lymph node ratios in node positive rectal cancer treated with preoperative chemoradiation. Asian Pac J Cancer Prev. 2013, 14:3769-72. 10.7314/APJCP.2013.14.6.3769

16. Junginger T, Goenner U, Lollert A, Hollemann D, Berres M, Blettner M: The prognostic value of lymph node ratio and updated TNM classification in rectal cancer patients with adequate versus inadequate lymph node dissection. Tech Coloproctol. 2014, 18:805-11. 10.1007/s10151-014-1136-X

17. Zeng WG, Zhou ZX, Wang Z, et al.: Lymph node ratio is an independent prognostic factor in node positive rectal cancer patients treated with preoperative chemoradiotherapy followed by curative resection. Asian Pac J Cancer Prev. 2014, 15:5365-9. 10.7314/APJCP.2014.15.13.5365

18. Koo T, Song C, Kim JS, et al.: Impact of lymph node ratio on oncologic outcomes in ypStage III rectal cancer patients treated with neoadjuvant chemoradiotherapy followed by total mesorectal excision, and postoperative adjuvant chemotherapy. PLoS One. 2015, 10:e0138728. Accessed: May 9, 2020: https://www.ncbi.nlm.nih.gov/pmc/articles/PMC4575157/. 10.1371/journal.pone.0138728

19. Park IJ, Yu CS, Lim SB, et al.: Ratio of metastatic lymph nodes is more important for rectal cancer patients treated with preoperative chemoradiotherapy. World J Gastroenterol. 2015, 21:3274-81. 10.3748/wjg.v21.i11.3274

20. Leonard D, Remue C, Abbes Orabi N, et al.: Lymph node ratio and surgical quality are strong prognostic factors of rectal cancer: results from a single referral centre. Colorectal Dis. 2016, 18:0175-84. 10.1111/codi.13362

21. Zuo ZG, Zhang XF, Wang H, et al.: Prognostic value of lymph node ratio in locally advanced rectal cancer patients after preoperative chemoradiotherapy followed by total mesorectal excision. Medicine (Baltimore). 2016, 95:e2988. 10.1097/MD.0000000000002988

22. Fritzmann J, Contin P, Reissfelder C, Büchler MW, Weitz J, Rahbari NN, Ulrich AB: Comparison of three 


\section{Cureus}

classifications for lymph node evaluation in patients undergoing total mesorectal excision for rectal cancer. Langenbecks Arch Surg. 2018, 403:451-62. 10.1007/s00423-018-1662-5

23. Chen L, Huang X, Song Z: The value of lymph node ratio in the prediction of rectal cancer patient survival after preoperative chemoradiotherapy. Int J Clin Exp Pathol. 2018, 11:5992-6001. Accessed: May 9, 2020: http://www.ijcep.com/files/ijcep0086231.pdf.

24. Vather R, Sammour T, Kahokehr A, Connolly A, Hill A: Quantitative lymph node evaluation as an independent marker of long-term prognosis in stage III rectal cancer. ANZ J Surg. 2011, 81:883-8. 10.1111/j.1445-2197.2010.05595.x

25. He WZ, Xie QK, Hu WM, et al.: An increased number of negative lymph nodes is associated with a higher immune response and longer survival in colon cancer patients. Cancer Manag Res. 2018, 10:1597-604. Accessed: May 9, 2020: https://www.ncbi.nlm.nih.gov/pmc/articles/PMC6014727/. 10.2147/CMAR.S160100

26. Fielding LP, Arsenault PA, Chapuis PH, et al.: Clinicopathological staging for colorectal cancer: An International Documentation System (IDS) and an International Comprehensive Anatomical Terminology (ICAT). J Gastroenterol Hepatol. 1991, 6:325-44. 10.1111/j.1440-1746.1991.tb00867.x

27. Scabini S, Montecucco F, Nencioni A, et al.: The effect of preoperative chemoradiotherapy on lymph nodes harvested in TME for rectal cancer. World J Surg Oncol. 2013, 11:292. Accessed: May 9, 2020: https://wjso.biomedcentral.com/articles/10.1186/1477-7819-11-292. 10.1186/1477-7819-11-292

28. Shimomura M, Ikeda S, Takakura Y, et al.: Adequate lymph node examination is essential to ensure the prognostic value of the lymph node ratio in patients with stage III colorectal cancer. Surg Today. 2011, 41:1370-9. 10.1007/s00595-010-4446-2

29. Zhang MR, Xie TH, Chi JL, et al.: Prognostic role of the lymph node ratio in node positive colorectal cancer: a meta-analysis. Oncotarget. 2016, 7:72898-907. Accessed: May 9, 2020:

https://www.ncbi.nlm.nih.gov/pmc/articles/PMC5341952/. 10.18632/oncotarget.12131

30. Chan DKH, Tan KK: Lower lymph node yield following neoadjuvant therapy for rectal cancer has no clinical significance. J Gastrointest Oncol. 2019, 10:42-7. 10.21037/jgo.2018.10.02 